\title{
Beitrag zur Kenntnis des Fettes vom Wasserhuhne (Fulica atra); der Grund des eigentümlichen Geruches und Geschmackes des Fleisches dieser Tiere.
}

\author{
Von
}

Tierarzt Dr. Sigismand Blaha.

(Aus dem Laboratorium für medizinische Chemie der Tierärztlichen Hochschule in Wien.) (Der Redaktion zugegangen am 13. Februar 1914.)

Das Wasserhuhn (Fulica atra), der bekannteste und verbreitetste Vertreter der Gattung «Wasserhühner \ (Fulica), findet auf unserem Markte als Wildbret nur wenig Beachtung. Es steht eben an Wohlgeschmack den anderen Sumpf- und Wasserhühnern bedeutend nach, was wohl hauptsächlich auf den dem Fleische und Fette anhaftenden eigentümlichen Geruch und Geschmack zurückzuführen ist. Wird es aber gehörig zugerichtet, in der Art, daß man vor der Zubereitung dem Tiere die Haut abzieht, so gibt es immerhin ein ganz annehmbares Gericht.

Es schien mir von Interesse, zu erfahren, was denn eigentlich der Grund des widerlichen Geruches und Geschmackes sei, der sehr an den der Sulzsardellen erinnert. In erster Linie wurde daran gedacht, daß eine besondere Beschaffenheit des Fettes den Grund für diese merkwürdige Eigenschaft des Fleisches bildet. Da in der Literatur nur eine einzige Untersuchung über das Fett vom Wasserhuhn zu finden ist, und zwar die von C. Schneider und S. Blumenfeld, ${ }^{1}$ ) so unternahm ich zunächst eine eingehende Untersuchung dieses Fettes, deren Resultate mit denen von Schneider und Blumenfeld nachher verglichen werden sollen.

Das Fett, welches zu meinen Untersuchungen diente, stammte zum Großteil aus dem Unterhautbindegewebe und dem

1) Beitrag zur Kenntnis animalischer Fette, Chemiker-Zeitung, 1906, Bd. 30, S. 53. 
Darmgekröse von ungefähr sechs Tieren. Dieses Gewebe wurde möglichst rein herauspräpariert, in der Porzellanschale auf ca. $30^{\circ} \mathrm{C}$. erwärmt und das ausgeschmolzene Fett mehrmals filtriert, wodurch die anhaftenden Gewebsteile entfernt wurden. Dann wurde es in einem verschlossenen Gefäße vor Licht und Luft . geschützt bei gleichmäßiger Temperatur aufbewahrt.

Schneider und Blumenfeld beschreiben das Fett folgendermaßen : hellgelbes, fast geruchloses, weiches Fett, welches sich nach einiger Zeit in einen weißen, festen und in einen gelben, flüssigen Anteil scheidet.

Bezüglich Farbe, Konsistenz und sonstigen Verhaltens des Fettes stimme ich mit Schneider und Blumenfeld überein; was aber den Geruch betrifft, so muß ich betonen, daß das Fett, wohl nicht so in dem Maße. als das Fleisch, ganz deutlich wahrnehmbar den eigentümlichen Geruch und Geschmack besitzt.

In dem von mir gewonnenen Fette bestimmte ich:
A. die Säurezahl,
B. die Verseifungszahl,
C. die Reichert-Meißlsche Zahl,
D. die Hehner-Zahl,
E. die Jodzahl des Fettes und der Fettsäuren,
F. die Acetylzahl der Fettsäuren,
G. das freie und das gebundene Cholesterin.

\section{A. Die Säurezahl.}

I. $5,4526 \mathrm{~g}$ Fett verbrauchten $10,2 \mathrm{ccm}$ Lauge $(1 \mathrm{ccm}=$ $0,00561 \mathrm{~g} \mathrm{KOH}$ ) entsprechend der Säurezahl 10,4 g.

II. $5,3808 \mathrm{~g}$ fett verbrauchten $9,8 \mathrm{ccm}$ Lauge $(1 \mathrm{ccm}=$ $0,00561 \mathrm{~g} \mathrm{KOH}$ ), entsprechend der Säurezabl 10,21.

\section{B. Die Verseifungszahl.}

I. 2,1474 g Fett verbrauchten nach der Verseifung mit $25 \mathrm{ccm}$ alkoholischer Kalilauge $8,9 \mathrm{ccm}$ Säure $(1 \mathrm{ccm}=31,751 \mathrm{mg}$ $\mathrm{KOH}) .25 \mathrm{ccm}$ alkoholische Kalilauge verbrauchten $22,0 \mathrm{ccm}$ derselben Säure, entsprechend der Verseifungszahl 193,8. 
II. $2,2778 \mathrm{~g}$ Fett verbrauchten nach der Verseifung mit $25 \mathrm{ccm}$ alkoholischer Kalilauge $8,2 \mathrm{ccm}$ Säure $(1 \mathrm{ccm}=31,751 \mathrm{mg}$ $\mathrm{KOH}) .25 \mathrm{ccm}$ alkoholische Kalilauge verbrauchten $22,1 \mathrm{ccm}$ derselben Säure, entsprechend der Verseifungszahl 193,8.

C. Die Reichert-Meißl sche Zàhl.

I. Die flüchtigen Fettsäuren aus 5,3622 g Fett verbrauchten 2,87 ccm 1/10-Normallauge, entsprechend der Reichert-Meißlschen Zahl 2,66.

II. Die flüchtigen Fettsäuren aus $5,221 \mathrm{~g}$ fett verbrauchten $2,391 / 10$-Normallauge, entsprechend der Reich ert-Meißlschen Zahl 2,29.

\section{Die Hehner-Zahl.}

I. 2,9687 g Fett lieferten 2,822 g Fettsäuren, entsprechend der Hehner-Zahl 95,1.

II. 2,960 g Fett lieferten 2,8215 g Fettsäuren, entsprechend der Hehner-Zahl 95,3.

\section{E. Die Jodzahl.}

I. $0,6278 \mathrm{~g} \mathrm{Fett}+50 \mathrm{ccm}$ Jodlösung verbrauchten 64,7 $\mathrm{ccm}$ Thiosulfat $(1 \mathrm{ccm}=0,0080 \mathrm{~g} \mathrm{Jod}), 50 \mathrm{ccm}$ Jodlösung verbrauchten 147,0 ccm Thiosulfat, entsprechend der Jodzahl 104,8.

II. $0,5712 \mathrm{~g}$ Fett. $+50 \mathrm{ccm}$ Jodlösung verbrauchten $71,9 \mathrm{ccm}$ Thiosulfat $(1 \mathrm{ccm}=0,0080 \mathrm{~g} \mathrm{Jod}), 50 \mathrm{ccm}$ Jodlösung verbrauchten 147,0 ccm Thiosulfat, entsprechend der Jodzahl 105,1.

\section{Jodzahl der Fettsäuren.}

Die Fettsäuren wurden in üblicher Weise aus dem Fette dargestellt.

I. $0,1551 \mathrm{~g}$ Fettsäure $+50 \mathrm{ccm}$ Jodlösung verbrauchten $73,9 \mathrm{ccm}$ Thiosulfat $(1 \mathrm{ccm}=0,0096 \mathrm{~g}$ Jod $), 50 \mathrm{ccm}$ Jodlösung verbrauchten $87,6 \mathrm{~cm}$ Thiosulfat, entsprechend der Jodzahl der Fettsäuren 84,5 .

!II. $0,3179 \cdot \mathrm{g}$ Fettsäure $+50 \mathrm{ccm}$ Jodlösung verbrauchten $63,2 \mathrm{ccm}$ Thiosulfat (1 $\mathrm{ccm}=0,0080 \mathrm{~g}$ Jod), $50 \mathrm{ccm}$ Jod- 
lösung verbrauchten $100 \mathrm{ccm}$ Thiosulfat, entsprechend der Jodzahl der Fettsäuren 92,6.

III. $0,2615 \mathrm{~g}$ Fettsäuren $+50 \mathrm{ccm}$ Jodlösung verbrauchten $84,9 \mathrm{ccm}$ Thiosulfat $(1 \mathrm{ccm}=0,0080 \mathrm{~g} \mathrm{Jod}), 50 \mathrm{ccm}$ Jodlösung verbrauchten $116,6 \mathrm{ccm}$ Thiosulfat, entsprechend der Jodzahl der Fettsäuren 96,9.

F. Die Acetylzahl der Fettsäuren.

Die aus dem Fette gewonnenen Fettsäuren wurden durch Kochen mit Essigsäureanhydrid in der üblichen Weise acetyliert.

I. 2;5512 $\mathrm{g}$ acetylierte Fettsäuren verbrauchten zur Neutralisation gegen Phenolphthalein $20,1 \mathrm{ccm}$ Lauge $(1 \mathrm{ccm}$ $=24,446 \mathrm{mg} \mathrm{KOH})$, entsprechend der Acetylsäurezahl 192,6, nach Zufügung von $29,9 \mathrm{ccm}$ derselben Lauge, somit im ganzen $50 \mathrm{ccm}$ Lauge, und Verseifung $21,4 \mathrm{ccm}$ Säure $(1 \mathrm{ccm}$ $=31,751 \mathrm{mg} \mathrm{KOH}$ ), entsprechend der Acetylverseifungszahl 212,7; daher ist die Acetylzahl 20,1.

II. 2,8938 g acetylierte Fettsäuren verbrauchten zur Neutralisation gegen Phenolphthalein $23,9 \mathrm{ccm}$ Lauge $(1 \mathrm{ccm}=$ 23,335 mg KOH), entsprechend der Acetylsäurezahl 192,7, nach Zufügung von 26,1 ccm derselben Lauge, somit im ganzen $50 \mathrm{ccm}$. Lauge, und Verseifung 17,4 ccm Säure $(1 \mathrm{ccm}=$ $31,751 \mathrm{mg} \mathrm{KOH}$ ), entsprechend der Acetylverseifungszahl 212,2; daher ist die Acetylzahl 19,5.

\section{Quantitative Bestimmung des Cholesterins.}

Zur quantitativen Bestimmung des Cholesterins nach A. Windaus ${ }^{1}$ ) wurden $2,8472 \mathrm{~g}$ Fett in der 50 fachen Menge kochenden Alkohols von $95 \%$ gelöst und mit einer $1 \%$ igen Lösung von Digitonin in heißem 90\% igen Alkohol versetzt. Das Gewicht dieses Niederschlages betrug 0,1165 g entsprechend 0,02832 $\mathrm{g}=0,99 \%$ Cholesterins (in freiem Zustande).

Zur Bestimmung des gebundenen Cholesterins wurde das Filtrat vom Digitonincholesterid vom Digitonin befreit; die Cholesterinester mit einer alkoholischen Lösung von Natriumäthylat

1) Diese Zeitschrift, Bd. 65, S. 110. 
auf dem Wasserbade verseift, das Cholesterin in der üblichen Weise durch mehrmaliges Ausschütteln mit Petroläther isoliert und nach der Digitoninmethode quantitativ bestimmt. Das Digitonincholesterid wog nach dem Waschen und Trocknen $0,0215 \mathrm{~g}$, entsprechend $0,00522 \mathrm{~g}=0,18 \%$ Cholesterin (zu Estern gebunden). Die Gesamtmenge des Cholesterins betrug demnach $1,17 \%$.

II. 2,9481 $\mathrm{g}$ Fett lieferten nach derselben Methode für freies Cholesterin 0,1356 g Digitonincholesterid, entsprechend $0,03296 \mathrm{~g}=1,12 \%$ Cholesterin, für gebundenes Cholesterin $0,0230 \mathrm{~g}$ Digitonincholesterid; entsprechend 0,0056 $\mathrm{g}=0,14 \%$ Cholesterin, zusammen 1,31\% Cholesterin.

Untersuchung des Muskelfettes.

Um einen Überblick zu gewinnen namentlich mit Rücksicht darauf, weil auch der Muskel charakteristischen Geruch und Geschmack zeigt, wurde auch das im Muskel enthaltene Fett einer Untersuchung unterzogen.

Ca. $80 \mathrm{~g}$ vom Fettgewebe tunlichst befreites Fleisch wurde faschiert und mit etwa der vierfachen Menge gebrannten Gipses verrieben; die trockene Masse wurde im Soxhletschen Apparate durch 3 Tage mit Äther extrahiert und der Äther verdunsten gelassen.

\section{A. Die Verseifungszahl.}

I. 1,4041 g Fett verbrauchten nach der Verseifung mit $25 \mathrm{ccm}$ alkoholischer Kalilauge 13,3 ccm Säure $(1 \mathrm{ccm}=31,751 \mathrm{mg}$ $\mathrm{KOH}), 25 \mathrm{ccm}$ alkoholische Kalilauge verbrauchten $21,7 \mathrm{ccm}$ derselben Säure, entsprechend der Verseifungszahl 189,9.

II. 2,4455 g Fett verbrauchten nach der Verseifung mit $25 \mathrm{ccm}$ alkoholischer Kalilauge 7,1 ccm derselben Säure, $25 \mathrm{ccm}$ alkoholischer Kalilauge verbrauchten 21,7 ccm derselben Säure, entsprechend der Verseifungszahl 189,5.

\section{B. Die Jodzahl.}

I. $0,2726 \mathrm{~g}$ Fett $+50 \mathrm{ccm}$ Jodlösung verbrauchten $49,0 \mathrm{ccm}$ Thiosulfat, $50 \mathrm{ccm}$ Jodlösung verbrauchten $81,4 \mathrm{ccm}$ Thiosulfat, entsprechend der Jodzahl 114. 
Beitrag zur Kenntnis des Fettes vom Wasserhuhne.

II. $0,3179 \mathrm{~g}$ Fett $+50 \mathrm{ccm}$ Jodlösung verbrauchten $63,2 \mathrm{ccm}$ Thiosulfat, $50 \mathrm{ccm}$ Jodlösung verbrauchten $100,0 \mathrm{ccm}$ Thiosulfat, entsprechend der Jodzahl 111.

Zur besseren Übersicht stelle ich meine Resultate für Fett aus Fettgewebe und für Fett aus Muskeln mit den Resultaten von Schneider und Blumenfeld in eine Tabelle zusammen.

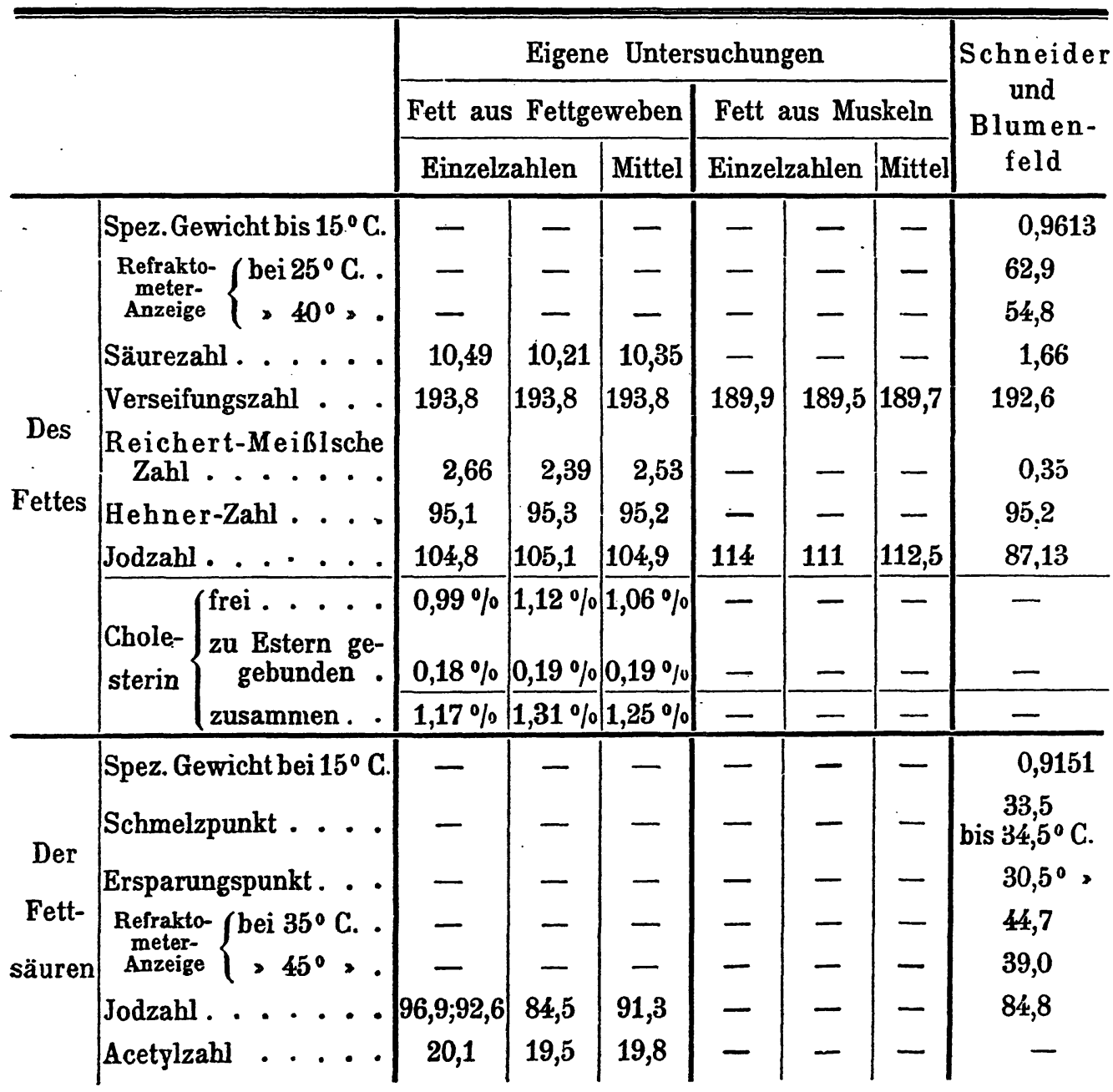

Im großen und ganzen betrachtet ist das Fett des Wasserhuhnes Fett von hoher Jodzahl und etwas höherer Säurezahl. Abgesehen davon weicht das Fett nach der Molekulargröße der Fettsäuren (Verseifungszahl, Reichert-Meißlsche Zahl,

Hoppe-Seyler's Zeitschrift f. physiol. Chemie. LXXXIX. 
Hehner-Zahl) dem Gehalte an Cholesterin und anderen Alkoholen (Acetylzahl) kaum von anderen tierischen Fetten ab.

Ist an sich eine hohe Jodzahl den Fetten der Vögel eigentümlich, so liegt die Jodzahl des Fettes vom Wasserhuhne noch erheblich höher, als es sonst bei Vögeln gewöhnlich ist. Das Fett des Wasserhuhnes nähert sich in dieser Beziehung den Fetten der Fische. Damit stimmt auch überein, daß die ungesättigten Säuren, sowie sie durch Verseifung des Fettes in Freiheit gesetzt worden sind, anscheinend recht leicht veränderlich sind; wenigstens glaube ich auf diesen Umstand die Tatsache zurückführen zu können, daß für die Jodzahl der Fettsäuren weder übereinstimmende, noch überhaupt plausible Zahlen erhalten werden konnten.

Daß das Muskelfett des Wasserhuhnes, soweit dies nachgewiesen werden konnte, nennenswert von dem Fette des Fettgewebes abweicht, bildet keine auffallende Erscheinung. Es ist dies ein ganz gewöhnlicher Befund und umsoweniger ins Gewicht fallend bei einem Tiere, bei welchem das Unterhautfettgewebe einen Wärmeregulator bei bedeutenden Temperaturschwankungen bilden muß.

Meine Untersuchungsresultate stimmen übrigens nur teilweise mit denen von Schneider und Blumenfeld überein. Ich finde die Säurezahl, die Jodzahl und Reichert-Meißlsche Zahl höher als Schneider und Blumenfeld, während die Hehner-Zahl und die Verseifungszahl so ziemlich übereinstimmen. Diese Differenzen erklären sich wohl aus der Verschiedenheit der Herkunft des Untersuchungsmaterials.

Schneider und Blumenfeld verarbeiteten Fett von Wasserhühnern, die aus der Skärengegend der Südküste Finnlands stammten. Die von mir untersuchten Wasserhühner sind in niederösterreichischen Gewässern erlegt und längere Zeit im Kühlraume im Gefrierzustand aufbewahrt worden. Bekanntlich hat das Klima, die Ernährung einen Einfluß auf die Zusammensetzung des Fettes, anderseits das Alter und die Art der Aufbewahrung eines Fettes einen Einfluß auf die Bildung von freien Fettsäuren.

Wir können daher folgenden Schluß ziehen: Der höhere 
Gehalt des Fettes an freien Fettsäuren ist wohl hauptsächlich auf die längere Aufbewahrung der Wasserhühner im Kühlraume zurückzuführen. Der größere Gehalt des Fettes an ungesättigten

Fettsäuren kann wohl bedingt sein durch die Art der Ernährung und durch den klimatischen Einfluß. Die größere Menge flüchtiger Fettsäuren endlich mag wohl darin ihren Grund haben, vielleicht aber auch von einer Zersetzung ungesättigter Säuren infolge langer Aufbewahrung herrühren.

Nach allem bisher Beschriebenen ergibt sich kein Anhaltspunkt dafür, daß der Grund für den eigentümlichen Geruch und Geschmack der Wasserhühner in der Beschaffenheit des Fettes zu suchen wäre, zumal da gerade jene Resultate meiner Untersuchungen, welche allenfalls in dieser Hinsicht verantwortlich gemacht werden könnten (Säurezahl, Reichert-Meißlsche Zahl) mit denen, welche von Schneider und Blumenfeld an anderswertigem Materiale gefunden worden sind, nicht übereinstimmen. Ich lenkte daher mein Augenmerk auf die Anwesenheit von flüchtigen Basen.

\section{Isolierung von Basen.}

Ca. $250 \mathrm{~g}$ faschiertes Fleisch wurde im Wasserdampfstrome der Destillation unterzogen, bis ungefähr 11 Destillat übergegangen war; letzteres wurde mit Äther ausgeschüttelt. Beide Teile, die Wasser- und Ätherflüssigkeit, wurden getrennt, mit wenig verdünnter Salzsäure versetzt und eingedampft. Der von der Ätherflüssigkeit erhaltene Abdampfrückstand war zu minimal, um weiter verarbeitet zu werden. Der Abdampfrückstand der wässerigen Lösung wurde in absolutem Alkohol gelöst. Das Filtrat abermals eingedampft und diese Behandlung solange wiederholt, bis kein Ammoniumchlorid ungelöst mehr zurückblieb. Hierauf wurde der Alkohol verdampft, der Abdampfrückstand in wenig Wasser gelöst und die wässerige Lösung mit Goldchloridlösung versetzt. Auch nach längerem Stehen hatte sich kein Niederschlag gebildet. Das Vorhandensein von Trimethylamin konnte dadurch ausgeschlossen werden.

Die goldchloridhaltige Flüssigkeit wurde in ein kleines Kölbchen gebracht und durch 12 Stunden mit Schwefelwasser- 
464 Sigismund Blaha, Zur Kenntnis des Fettes vom Wasserhuhne.

stoffgas zur Entfernung des Goldes behandelt. Vom abgeschiedenen Goldsulfid wurde abfiltriert, das Filtrat verdampft, der Abdampfrückstand in wenig absolutem Alkohol aufgenommen und die Lösung mit Platinchlorid versetzt. Es schied sich ein gelber, krystallinischer Niederschlag aus, der für die Anwesenheit von Methylamin sprach, da das Platindoppelsalz des Dimethylamins zwar im kalten Wasser schwer löslich, im Alkohol aber leicht löslich ist.

Der gelbliche Niederschlag wurde auf einem Filter gesammelt, getrocknet und mit einer Federfahne in einen Tiegel gebracht, gewogen, hierauf geglüht und nach dem Glühen wieder gewogen.

$0,0219 \mathrm{~g}$ des trockenen Niederschlages hinterließen beim Glühen $0,0090 \mathrm{~g} \mathrm{Pt}$, entsprechend 41,1\% Pt (berechnet für $\mathrm{C}_{2} \mathrm{H}_{12} \mathrm{~N}_{2} \mathrm{PtCl}_{6}: 41,3 \% \mathrm{Pt}$ ). Da aus $250 \mathrm{~g}$ Fleisch $0,0214 \mathrm{~g}$ Methylaminplatinchlorid, entsprechend $0,00288 \mathrm{~g}$ Methylamin, erhalten worden sind, so würden auf $1 \mathrm{~kg}$ Fleisch, die unvermeidlichen Verluste nicht gerechnet, $12 \mathrm{mg}$ Methylamin kommen.

Da der Geruch des Fleisches der Wasserhühner nicht wesentlich von dem des Methylamins abweicht, so kann der Geruch des Fleisches der Wasserhühner der Hauptsache nach auf die Anwesenheit von Monomethylamin zurückgeführt werden. 